\title{
L'Esthétique du jeu cruel dans la tragédie domestique élisabéthaine et jacobéenne
}

Jean-Claude Mailhol

\section{(2) OpenEdition}

\section{Journals}

Édition électronique

URL : http://journals.openedition.org/shakespeare/657

DOI : 10.4000/shakespeare.657

ISSN : 2271-6424

Éditeur

Société Française Shakespeare

Édition imprimée

Date de publication : 1 novembre 2005

Pagination : 91-107

ISBN : 2-9521475-2-3

\section{Référence électronique}

Jean-Claude Mailhol, «L'Esthétique du jeu cruel dans la tragédie domestique élisabéthaine et

jacobéenne », Actes des congrès de la Société française Shakespeare [En ligne], 23 | 2005, mis en ligne le 01 janvier 2007, consulté le 19 avril 2019. URL : http://journals.openedition.org/shakespeare/657 ;

DOI : 10.4000/shakespeare.657 


\section{Shakespeare et le jeu}

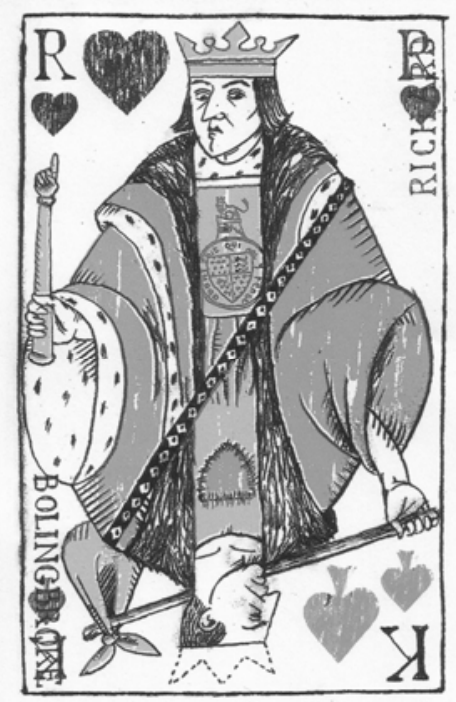

actes du Congrès organisé par la

SOCIÉTÉ FRANÇAISE SHAKESPEARE les 10, 11 et 12 mars 2005

textes réunis par Pierre KAPITANIAK sous la direction de Yves PEYRÉ 


\title{
COMITÉ SCIENTIFIQUE :
}

\author{
Margaret Jones-Davies \\ Gisèle Venet \\ Jean-Marie Maguin \\ Yves Peyré \\ François Laroque \\ Pierre Kapitaniak
}

COUVERTURE :

Edouard Lekston, King and Kusin, 2005

edlek@free.fr

conception graphique et logo

Pierre Kapitaniak

(C) 2005 Société Française Shakespeare

Institut du Monde Anglophone

Université de Paris III - Sorbonne Nouvelle

http://recherche.univ-montp3.fr/SFS/

5 rue de l'École de Médecine

75006 Paris

ISBN 2-9521475-2-3

Tous droits de traduction, de reproduction et d'adaptation réservés pour tous les pays 


\title{
L'ESTHÉTIQUE DU JEU CRUEL DANS LA TRAGÉDIE DOMESTIQUE ÉLIS ABÉTH AINE ET JACOBÉENNE
}

\author{
Jean-Claude MAILHOL
}

\begin{abstract}
Dans cette communication seront passées en revue les différentes formes de jeu présentes dans la tragédie domestique : jeu de cartes, jeu de dés, jeu de trictrac, jeu des armes, jeu de chasse. Certaines activités ludiques étaient parfois connotées négativement. Ainsi la figure du « fils prodigue ", possédé par le démon du jeu, devient récurrente dans le drame domestique : elle s'incarne dans le sinistre héros de $A$ Yorkshire Tragedy, jouet d'un destin qu'il ne contrôle plus. II est victime du jeu, cause de sa perdition et de celle de sa famille. Le personnage et l'accessoire scénique symbolisant sa déchéance, le dé, ne font plus qu'un. Le lexique, mais aussi le jeu de l'acteur, reflètent la condition du héros damné. A travers le jeu de société se déploie tout un jeu verbal où le sens des mots se dédouble. Le jeu de cartes devient jeu sexuel dans la tragédie de Heywood $A$ Woman Killed with Kindness, alors que, dans la grande tragédie anonyme Arden of Faversham, le jeu de dés permet aux personnages malfaisants de jouer avec la vie de leurs semblables. Mais le jeu n'est-il pas avant tout d'ordre esthétique ? Certes, dans le jeu de la chasse sont mises en scène les relations entre dominant chasseur et proie chassée, comme dans Two Tragedies in One. Mais le dramaturge développe parallèlement une esthétique poétique : Robert Yarington joue des échos et des effets de miroir, par l'introduction de doubles. Mis en abyme dans une scène, le motif de la chasse se mue alors en une ample métaphore où le jeu de création artistique n'est pas incompatible avec une vision sombre du monde.
\end{abstract}

This paper examines the various types of games to be found in domestic tragedy : cards, dice, backgammon (tables), arms games, hunting game. Some play activities could be fraught with negative connotations. For instance the figure of the "prodigal son ", possessed by his passion for gambling, becomes recurrent in domestic drama. It is embodied in the gruesome hero of A Yorkshire Tragedy who is the plaything of an uncontrollable fate, and whose gaming passion brings about his and his family's ruin. As the drama unfolds, the spectator witnesses the merging of the character and the stage property of the dice which is the symbol of his downfall. The vocabulary and the actor's performance mirror the damned character's plight. With parlour games is triggered off some verbal playing with double meaning puns. In A Woman Killed with Kindness, the cards game becomes sexual while in the great anonymous tragedy, Arden of Faversham, dice playing enables the wicked characters to sport with the hero's life. The game is also an aesthetic one. With hunting are staged the relationships between the dominating hunter and the hunted prey, as is the case in Two Tragedies in One. But at the same time the playwright, Robert Yarington, develops a poetic and aesthetic strategy as he plays with echoes and mirror effects by introducing doubles. The hunting motif is inset in a scene, and then becomes a pervasive metaphor in the tragedy where artistic creation results in a gloomy vision of the world.

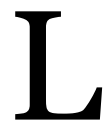
es personnages de la tragédie anonyme Arden of Faversham se sont réunis entre intimes avant le repas, sous le toit domestique de la demeure d'Arden, dans le Kent, prêts à goûter un moment de détente, tandis que ceux de la tragédie de Thomas Heywood, $A$ Woman Killed with Kindness, engagent les paris pendant qu'ils s'adonnent dans la campagne à la pratique de la fauconnerie, activité réservée non seulement à l'aristocratie, mais aux nantis de la classe 
moyenne. Le jeu, composante culturelle observable dans toute société, touche tous les milieux. Mais qui dit jeu dit aussi compétition et lutte. Chacun connaît les règles du jeu social codifié, mais, derrière le jeu se cachent d'autres enjeux, souvent sinistres et morbides. On ne saurait oublier que les tragédies domestiques ${ }^{1}$ sont aussi connues sous le nom de "murder plays ", et dans ce petit monde en apparence paisible où "tous se livrent à de vertueuses et profitables affaires" (Two Lamentable Tragedies, i.6) rôde le spectre de la mort violente.

Dans cette étude seront analysées plusieurs formes de jeu. Simple divertissement verbal au départ, l'activité ludique peut se transformer en jeu pervers révélateur de la corruption des âmes. Ensuite on s'intéressera au jeu de dés, thématique déjà présente dans les moralités, reprise dans le théâtre domestique qui en fait une métaphore de la confusion. Enfin, le jeu des armes et, en particulier, le jeu de chasse constituent un contexte. Les dramaturges l'exploitent à un niveau métaphorique pour livrer la vision globale d'un monde implacable où les relations se construisent sur le mode de la prédation.

\section{Divertissements coupables et jeux de langage dévoyés}

Dans A Woman Killed with Kindness, l'annonce de l'infidélité d'Anne amène Frankford à réunir quatre personnages autour de la table pour jouer aux cartes :

FRANKFORD. Lights and a table there! Wife, Master Wendoll and gentle Master Cranwell -

Enter ANNe, Master Wendoll, Master Cranwell, Nicholas and JENKINS, with cards, carpet, stools, and other necessaries.

(iii.116-17)

\footnotetext{
${ }^{1}$ Éditions des tragédies domestiques analysées :

- Arden of Faversham (anon.), 1592, éd. Martin White, coll. The New Mermaids, Londres, A. \& C. Black, (1982) 1995.

- A Yorkshire Tragedy (anon.), 1605, éd. A.C. Cawley and Barry Gaines, coll. The Revels Plays, Manchester University Press, 1986.

- Thomas Heywood, A Wom an Killed with Kindness, 1607, éd. Brian Scobie, coll. The New Mermaids, Londres, A. \& C. Black, (1985) 1991.

- Robert Yarington, Two Lamentable Tragedies (Two Tragedies in One), 1594-1595, 1601, dans A Critical Old-Spelling Edition of Robert Yarington's «Two Lamentable Tragedies», éd. Anne Weston Patenaude, Ann Arbor, Michigan (1978), University Microfilms International Dissertation Services, 1997.
} 
La situation d'adultère a fait basculer l'héroïne dans un univers de perversion, traduit par une corruption du langage employé dans la scène de la partie de cartes où participent notamment le mari, la femme et l'amant (viii.118-197). Le jeu, fortement connoté à l'époque élisabéthaine et jacobéenne, suggère le dérèglement, les différentes formes de jeu étant généralement jugées suspectes et malsaines. Les puritains étaient les premiers à les condamner. Stephen Gosson écrit : « Dicers and carders because their abuses are commonly cryed out on, as usually shewen, have no neede of a needelesse discourse, for every manne seeth them, and they stinke almost in every mans nose ${ }^{2}$. » Philip Stubbs tolère le jeu uniquement dans la situation exceptionnelle où les individus s'y adonnent avec modération pour se changer les idées et se délasser après une activité cérébrale importante : «... for their privat recreations, after some oppression of studie, to drive fantasies, and such like, I doubt not, but they may, using it moderatly, with intermission, and in the feare of God ${ }^{3}$. » Bien sûr, Frankford n'est pas du tout dans cette situation, et la crainte de Dieu n'effleure pas un instant non plus son esprit, tout occupé à chercher le moyen de confondre les amants coupables par la ruse :

FRANKFORD. Distraction I will banish from my brow, And from my looks exile sad discontent.

Their wonted favours in my tongue shall flow.

Till I know all, I'll nothing seem to know.

(viii.112-15)

L'apparence triomphe, car même le mari trompé est amené à jouer sournoisement un rôle de composition, comme le suggère la présence du verbe «seem » dans la bouche de Frankford (v. 115). La langue corrompue déverse le mensonge (v. 114).

La dépravation verbale se traduit littérairement et linguistiquement par un dédoublement du sens des mots. Le vocabulaire exprimant la notion de double surgit immédiatement dans

\footnotetext{
${ }^{2}$ Stephen Gosson, The Schoole of Abuse, 1579, éd. Arthur Freeman, réimpression de facsimilé, coll. Garland Publishing, New York, 1973, sig. D4r ${ }^{\circ}$. Voir aussi Robert Burton, The Anatomy of Melancholy, 1621, pt. 1, sec. 2, mem. 3, subs. 13, "Love of Gaming, etc., and Pleasures Immoderate, Causes », p. 287-292.

3 Philip Stubbs, The Anatomy of Abuses, 1583, STC, réimpression de fac-similé, coll. Johnson Reprint Corporation, New York, 1972, sig. O7r ${ }^{\circ}$.
} 
le lexique des joueurs et même des spectateurs de la scène : "pair of cards » (v. 121), «Double Ruff» (v. 143), « double hand» (v. 145), «the grossest pair» (v. 173). Mais surtout plusieurs mots sont à double sens, dont le premier est "match» (v. 129 et 130). Il vient naturellement en réponse à la question provocante d'Anne qui, au début de la scène, cherche son "partenaire»: "Come, Master Frankford, who shall take my part? » (v. 126). Cette question donne le ton d'emblée. Elle est propice à déclencher un dérapage générateur d'une confusion sémantique, entretenue dans le dialogue qui va suivre, par chacun des intervenants complaisants. Le verbe " take », terme de jeu, a donc un double sens. Frankford demande à Cranwell : « shall you and I take them up ?» (v. 132-133). Les autres personnages brodent autour du verbe «take »; il assume alors des sens différents, souvent connotés sexuellement (v. 138, 139, 150, 181). Des termes comme «take» ou «match » se prêtent à une double signification. Le sens immédiat du mot «match » désigne le partenaire au jeu de cartes, et, dans le contexte de la scène, le terme désigne d'abord le partenaire du jeu sexuel. Wendoll utilise le mot le premier (v. 129), puis Frankford le reprend (v. 130). Ainsi l'amant et le mari sont en concurrence, l'épouse ayant provoqué verbalement cette rivalité (v.126) dont elle va être l'arbitre. Le mot "match » avait déjà été employé au tout début de la pièce ${ }^{4}$. À l'ouverture de l'œuvre, Frank et Anne semblent être un couple de jeunes mariés parfaitement assortis: «... and your hands / Methinks are matches»(i.65-66), leur dit Sir Charles. Le sens du terme se dévoie lorsque, dans la même scène, Charles propose à Francis de participer à une chasse au faucon : "I'll make a match with you : meet me tomorrow / At Chevy Chase, I'll fly my hawk with yours" (i.93-94). Tout de suite interviennent les notions de compétition puis d'argent, avec l'introduction de l'enjeu, au moment du pari entre Sir Charles et Sir Francis. L'interlocuteur accepte la proposition: "'Tis a match, 'tis done» (v. 98). Lorsque le terme réapparaît à la huitième scène, le contexte laisse entendre que l'amant a déjà l'ambition d'évincer le mari. Celui-ci commente, avec une amertume impuissante, le partenariat de Wendoll et d'Anne autour de

\footnotetext{
${ }^{4}$ A Woman Killed with Kindness, op. cit. p. 44, n. 129.
} 
la table de jeu : «I do not like that match » $(8,130)$. John se perçoit déjà exclu de ce couple heureux formé autrefois avec Anne. Le lexique disait l'harmonie d'antan; il reste inchangé, mais se pervertit en désignant une nouvelle réalité grinçante. D’autres termes sont à double entente: "playing fake» (v. 136 et 138)5, "playing false » (v. 136), " heart » (v. 187 et 189), et à cette liste s'ajoute l'homophonie courante à l'époque élisabéthaine entre « queen » (v. 171) et « quean» (v. 172) Tout le vocabulaire gravitant autour du thème du jeu de cartes est systématiquement chargé d'allusions sexuelles obscènes : "Noddy » (v. 141), «Double Ruff» (v. 143)7, « new cut» (v. 153) ${ }^{8}$, « Post and Pair » (v. 160), « deal » (v. 167) et « dealt » (v. 174, 182, 183 et 193) 9.

\section{Le jeu de dés : de la moralité à la tragédie domestique}

Les scènes de jeu de dés occupaient aussi une place non négligeable dans le théâtre élisabéthain. Bien sûr le jeu de dés faisait partie de la liste des vices condamnés entre tous par les puritains, mais les défenseurs du théâtre s'empressèrent de démontrer que, si la thématique du jeu de dés était mise en scène, c'était bien sûr dans un but louable de pédagogie morale. Le « water poet » John Taylor écrivit dans la sixième dédicace du célèbre ouvrage de Thomas Heywood, $A n$ Apology for Actors :

A play's a true transparant christall mirror, To shew good minds their mirth, the bad their terror : Where stabbing, drabbing, dicing, drinking, swearing, Are all proclaim'd unto the sight and hearing,

\footnotetext{
5 Claude Rudigoz, Systématique génétique de la métaphore sexuelle, Lille, Atelier de reproduction des thèses, 1978, p. 1132.

${ }^{6}$ Ibid., p. 1250.

${ }^{7}$ Rudigoz, op. cit., p. 1258.

8 Ibid., p. 1114 et 1137.

${ }^{9}$ Ibid., p. 1117. Pour tous ces termes, on peut aussi consulter les notes de l'édition de B. Scobie, p. 44-48, et celles de l'édition de R. W. Van Fossen, éd. Methuen \& Co, coll. The Revels Plays, Londres, 1961, p. 49-53.
} 
In ugly shapes of Heaven-abhorrid sinne,

Where men may see the mire they wallow in ${ }^{10}$.

Dans ces trois paires rimées de vers, le jeu de dés figure parmi les cinq abominations les plus monstrueuses, réunies et soulignées par les homéotéleutes en [I]], issus de l'accumulation des noms verbaux (v. 11). Le théâtre, selon Heywood (et Taylor), se devait de dénoncer de tels vices par la représentation de ces mêmes vices sur les planches, dans un but dissuasif.

Si le drame domestique s'intéresse à la thématique du jeu de dés, avec la mise en scène de la figure du « fils prodigue», on n'oubliera pas l'influence déterminante des moralités qui le précéda. Elles esquissent déjà le personnage du noceur passant les nuits de sa jeunesse déréglée dans les tavernes. Devenu «Lust and Liking » dans Mundus et Infans ${ }^{11}$, le héros s'adonne à la fête et au plaisir du jeu : "All game and glee, / ... / All revel and riot» (v. 140-142). " Husband », héros de la tragédie domestique A Yorkshire Tragedy, dont le patronyme générique fait penser aux personnages des moralités, ressemble aux «prodigals » qui font la fête dans The Disobedient Child ${ }^{12}$ (v.539-554), Everyman ${ }^{13}$ (v. 272-273), The Interlude of Youth ${ }^{14}$ (v. 283-288, v.375-376, v.399-401) et Nice Wanton $^{15}$ (v. 68-70). Dans cette dernière œuvre, « Wordly Shame» se réjouit de la vie de débauche menée par Ismael et Dalilah (v. 446-450). Dans Mundus et Infans, "Folly » entraîne «Manhood » dans les tavernes louches du quartier londonien d'Eastcheap (v. 671). Là,

\footnotetext{
10 John Taylor, «To my approved good friend M. Thomas Heywood », dans Thomas Heywood's An Apology for Actors, 1608-1612, éd. Arthur Freeman, New York, Garland Publishing, 1973, sig. A7v ${ }^{\circ}$, v. 9-14.

${ }^{11}$ Mundus et Infans, dans Three Late Medieval Morality Plays, éd. G. A. Lester, coll. The New Mermaids, Ernest Benn, Londres, 1981, p. 108-157.

${ }^{12}$ Thom as Ingelend, The Disobedient Child, environ 1549, dans The Dramatic Writings of Richard Wever and Thom as Ingelend, éd. John S. Farmer, coll. Early English Dramatists, Early English Drama Society, Londres, (1905) Rpt Charles W. Traylen, Guilford, 1966, p. 43-92.

13 Everyman, dans Three Late Medieval Morality Plays, op. cit., p. 59-105.

14 The Interlude of Youth, 1513-1514, dans Two Tudor Interludes, éd. Ian Lancashire, coll. The Revels Plays, Manchester University Press, 1980, p. 100-152.

15 Nice Wanton, dans English Moral Interludes, éd. Glynne Wickam, Londres, 1976, p. 143162.
} 
«Folly» propose un jeu de dés, appelé «passage ${ }^{16}$. " Dans Lusty Iunentus ${ }^{17}$, «Iuuentus », «prodigal » futile, suit « Felowshyp », « Hypocrisie » et «Abhominable Liuing ». « Good Councell » est désespéré :

Good COUNCELL. But now he walketh, alas : in the vngodlies chase,

Heaping sinne vpon sinne vyce vpon vyce,

He that liueth most vngodly is counted most wyse.

\section{Here entreth Iuuentus.}

IUUENTUS. Who is here playing at the dice?

I heard one speake of synnes and sice ${ }^{18}$

His wordes did me entice,

Hither to come.

(v. 936-942)

Entré dans l'engrenage de la «chaîne du vice ${ }^{19}$ » (v. 937), le personnage, comme «Husband» dans A Yorkshire Tragedy, est possédé par le démon du jeu. La rime réunit les quatre termes, « vyce » (v 937), « Dice » (v. 939), « sice » (v. 940) et « entice» (v. 941). Tous ces lexèmes se terminent sur le phonème alvéolaire fricatif sourd [s], souvent métaphoriquement évocateur du sifflement du serpent, associé au diable. Les quatre rimes encadrent, par contraste, le dernier mot du vers 938, l'adjectif « wise », concluant la tirade de « Good Councell ». Ainsi, le dernier mot de chacun des cinq vers (v. 937-941) comporte la diphtongue [aI], mais l'adjonction du phonème fricatif qui suit la diphtongue ([s] pour « vyce », « Dice », « sice » et « entice », [z] pour « wyse »), en introduisant l'opposition distinctive des traits [sourd] / [sonore], contribue à créer une différenciation qui n'est plus seulement phonétique, mais sémantique, à l'intérieur des paires minimales de

\footnotetext{
${ }^{16} \mathrm{Ce}$ jeu nécessitait l'usage de trois dés. Le terme «passage » était métonymiquement devenu synonyme de « dice ». Voir A.C. Cawley et Barry Gaines, Introduction et notes de $A$ Yorkshire Tragedy, p. 76, note 65 .

${ }^{17}$ Richard Wever, An Enterlude Called Lusty Iuuentus, dans An Enterlude Called Lusty Iuuentus: An Old-Spelling Critical Edition, éd. H. S. Thomas, coll. The Renaissance Imagination, vol. 2, Garland Publishing, New York, 1982.

${ }^{18}$ L'expression «synnes and sice » dérive, par un jeu de mots provenant du français, de " cinq et six », qui, au jeu de dés, correspondent aux deux faces totalisant le plus grand nombre de points. Voir Helen Scarborough Thomas, Introduction et notes de An Enterlude Called Lusty Iunentus, op. cit., p. 81-82, note 940.

19 Voir Henry Hitch Adams, English Domestic or, Homiletic Tragedy: 1775 to 1642, Columbia University Press, New York, 1943, p. 118-119.
} 
termes. À la sagesse de «Good Councell » (v. 938), s'oppose donc la perversion vicieuse et sournoise du «prodigal » (v. 937, 939, 940 et 941). Possédé par le diable, «Iuuentus » joue aux dés. Dans Nice Wanton, "Iniquity » jette les dés, signe ostensible que les deux personnages vont sombrer dans le mal (v. 172, didascalie : He casteth dice on the board). Leur perdition se cristallise dans la scène du jeu de dés (v. 210-219), où sont évoqués le diable (v. 219) et les enfers (v. 222). Le «prodigal», Ismael, comprend trop tard qu'il a été la victime du mal. L'activité du jeu de dés était le signe du début de sa chute, exprimant l'emprise du diable sur lui : " His naughty company and play at dice / Did me first to stealing entice » (v. 400-401). Comme dans la moralité, la compagnie du diable a eu un effet néfaste sur « Husband » : « O, 'twas the enemy my eyes so bleared ! ( (x.46). Mais, à la différence des moralités, l'auteur de $A$ Yorkshire Tragedy ne met pas directement en relation la thématique du jeu de dés avec celle du diable. Il les sépare, en faisant du personnage principal un " prodigal », fêtard nocturne, et, par ailleurs, sournoisement habité par le Malin.

Dans Homo Ludens (1938), Johan Huizinga montre que toutes les activités humaines relèvent de l'instinct du jeu, élément culturel fondamental. Cet instinct de jeu s'illustre de façon éclatante, à différents niveaux, dans la grande tragédie domestique Arden of Faversham, d'abord dans le jeu de dés. La métaphore du jeu devient ensuite obsédante dans l'œuvre. La première référence à la paire de dés arrive très tôt :

ALICE. Bear him from me these pair of silver dice With which we played for kisses many a time, And when I lost I won, and so did heSuch winning and such losing Jove send me !

L'amour, associé au jeu de hasard (v. 125-126), apparait alors, plus comme une activité ludique (v. 124), que comme une valeur permanente. Les dés sont associés au jeu amoureux, et Alice rappelle à Mosby leur complicité dans ces moments de divertissement où, jouant avec les dés d'argent, les amants entretenaient l'illusion d'être à l'unisson. Le vocabulaire antithétique va aussi dans ce sens («lost» / «won », v. 125, et « winning » / « losing », v. 126), et il fait peut-être 
surgir, dans la mémoire du spectateur, le souvenir de la très ancienne thématique héritée de l'antiquité païenne ${ }^{20}$, faisant de la Fortune capricieuse une figure allégorique. Selon son gré, elle rendait l'individu, tantôt gagnant, tantôt perdant ${ }^{21}$. Alice se place résolument dans une perspective plane, excluant à dessein tout désir de transcendance, en niant l'existence du dieu chrétien, puisque le personnage affiche des références païennes au travers d'une allusion à Jupiter («Jove», v. 126). Un peu plus loin, Mosby fait sa première apparition sur scène (v. 175, didascalie, et v. 176), en tenant certainement à la main la paire de dés ${ }^{22}$, don de la jeune femme.

On peut rapprocher la thématique du dé dans Arden of Faversham et dans A Yorkshire Tragedy, où le destin du héros, figure de « fils prodigue », dépend des aléas du jeu. Le vocabulaire en relation avec le jeu de dés est d'abord employé au sens propre (ii.7), avant de devenir symbolique de la destinée hasardeuse du personnage. En proie au démon du jeu, "Husband» met aussi en péril sa propre descendance (iv.64-68). Comme dans Arden of Faversham, l'auteur introduit le substantif « dice », qu'il fait cohabiter avec les deux termes « lost» et «won », en associant le dernier au passé prestigieux de la famille, et en l'opposant en même temps au premier, devenu synonyme de déchéance : "'Tis lost at dice what ancient honour won: / Hard when the father plays away the son » (v.4-5). Au fur et à mesure que l'action évolue, le héros, inconscient d'être le jouet de son vice, devient assimilable à ce dé que le hasard fait rouler à sa guise. Comme dans les moralités, l'accessoire du dé fait partie de la panoplie du « fils prodigue », mais il devient ensuite un moyen littéraire utilisé par le dramaturge, lorsque le héros commente son infortune. « Husband », venant de perdre à une partie de dés, fit sa première apparition sur scène en s'écriant: "Pox o'th'last throw!" (ii.25). À la fin de la tragédie, «Husband» tombe de cheval, au moment de sa fuite. Le

\footnotetext{
${ }^{20}$ Willard Farnham, The Medieval Heritage of Elizabethan Tragedy, Oxford, Basil Blackwell, (1936) 1963, chap. 1 « Greco-Roman Surrender of the World », p. 3-5 et p. 27-29. ${ }^{21}$ Dans King Lear, Cordelia, vaincue, évoque la Fortune contraire («false fortune's frown », v.iii.6), tandis que Lear rappelle le caractère incertain de toute chose, si bien que chacun peut autant perdre que gagner : « Who loses and who wins, who's in, who's out... » (V.iii.15).

22 Dans son édition de la pièce, Martin White suggère l'idée, p. 11, note 184.
} 
comédien incarnant «Husband » projeté à terre, roule sur la scène ouverte. Le personnage se confond alors, en raison du lexique choisi, avec un dé qui a été jeté : «...Heart! of chance / To throw me now within a flight o'th'town... » (viii.4-5). Le personnage poursuit ses allusions au jeu de dés : «'Sfoot, a man / May dice upon't... » (v. 6-7). Le motif du dé se pare donc d'une dimension esthétique qu'il n'avait pas dans les moralités. Il devient une métaphore théâtrale mettant en étroite relation le héros avec le dé. Par le jeu de la synecdoque théâtrale, le héros et le dé ne font plus qu'un.

Dans Arden of Faversham, le motif du dé en mouvement est en corrélation avec la notion de changement, et il suggère le caractère incertain et versatile des choses. Le motif du dé qui roule, qui va et qui vient, est une métaphore théâtrale rendant compte de la relation amoureuse instable (i.123-126). Mais, de manière plus globale, le mouvement de va-et-vient constitue un véritable motif esthétique présidant à l'unité de la tragédie. Dès le début, l'allusion aux lettres d'amour, échangées par les amants, sert de prélude à ce mouvement de « navette » qui ne cessera de traverser la tragédie domestique. Arden souligne le caractère perverti de ce mouvement de navette épistolaire, installée entre Alice et Mosby : « Love letters pass 'twixt Mosby and my wife... » (i.15). D'entrée, le spectateur sait que le héros est au courant de l'adultère de sa femme, dont il souffre (v. 10-11). Mais ce tourment est à la source d'une autre forme d'instabilité venant affecter le héros. Le fonctionnement pervers d'Alice conditionne, par un phénomène de ricochet, le comportement d'Arden. À son tour, le héros devient sujet à l'instabilité. Arden ne connait plus de tranquillité dans son propre foyer, rempli de la présence de l'amant. Ballotté comme le dé, Arden ne peut plus rester en place (i.54-55, 81, 92, 299, 399, 408, 416 ; vi.41-44 ; x.34). Les errances du personnage, condamné à ne plus rester en place, sont une marque d'agitation et d'instabilité. Ils reflètent le tourment du héros, incapable de trouver l'apaisement, et en proie à la plus grande confusion $^{23}$. Arden est ballotté comme le dé, image qui se répercute

${ }^{23}$ L'incapacité du personnage à rester en place a été interprétée aussi en termes socioéconomiques. Ann Christensen montre que les héros des tragédies domestiques, appartenant à la classe moyenne émergente du début de l'ère moderne capitaliste, sont en proie à une inquiétude liée à leurs obligations professionnelles de se déplacer : "They are 
dans les métaphores du retournement, du revirement, et, de façon plus générale, dans toutes celles qui mettent en jeu une dynamique d'opposition. À la fin de la pièce, la symbolique théâtrale de la paire de dés, introduite à l'autre bout de la tragédie (i.123-126 et 215-216), surgit à nouveau (xiv.222-232)24. Mais, les dés, associés au jeu amoureux hasardeux dans la première scène, font une nouvelle apparition ici pour renseigner sur le caractère tout particulier de la partie qui va se jouer, et dont l'enjeu véritable réside dans la mise à mort du mari gênant. À Mosby revient l'idée du jeu de dés : " I'll fetch Master Arden home, and we, like friends, / Will play a game or two at tables here » (xiv.98-99). Mosby et Arden s'installent donc autour de la table, au centre de la scène ouverte. Mosby s'impose comme le maître du jeu, en prenant l'initiative de jeter un premier dé (v. 230, didascalie). Mais, ironiquement, ce jeu n'a rien de spontané ni d'impromptu. Pour une fois, aucune place n'est laissée au hasard, puisque le jeu de dés n'est que le prétexte permettant d'introduire le mot d'ordre convenu plus tôt (v. 104) par les intrigants : « Now I can take you » (v. 232). Dans leur jeu amoureux instable, Alice et Mosby se divertissaient, tantôt à gagner, tantôt à perdre (i.125-127). Inversement, à la quatorzième scène, Arden est le perdant désigné d'une fausse partie de dés, prélude macabre d'une mort soigneusement organisée. L'accessoire de la paire de dés permet donc de dynamiser une métaphore théâtrale, suggérant que les deux amants diaboliques ont joué avec la destinée d'Arden. Le héros perd la vie au cours de la partie de trictrac.

householders, landowners, and merchants, whose domiciles fall vulnerable to 'invasion and adultery', often from within, while the heroes themselves are away on some business. » Elle établit un parallèle entre les héros masculins des diverses tragédies domestiques, partagés entre le foyer et le monde des affaires, et incapables de trouver une harmonie entre les deux. A. Christensen, «Business, Pleasure and Domestic Economy in Heywood's A Woman Killed with Kindness ", Exemplaria : a Journal of Theory in Medieval and Renaissance Studies, vol. 9, fasc. 2, septembre 1997, p. 315-340, p. 322.

24 Dans son édition d'Arden, Martin White formule l'hypothèse que la même paire de dés, utilisée comme accessoire scénique dans la première scène, sert à nouveau à la quatorzième scène: "Presumably, it should be these same dice with which Mosby and Arden play in Scene xiv. » (p. 11, note 184). 
Tout au long de la pièce, Arden va et vient, entre et sort. Ce mouvement incessant de navette, absurde et désordonné ${ }^{25}$, se réduit à une agitation reflétant la situation d'un être inquiet et perturbé, qui a perdu ses repères et ne maîtrise pas l'objet de ses déplacements. La vie d'Arden est soumise aux aléas du hasard comme l'est une partie de dés. La succession des incidents insignifiants, des multiples tentatives avortées d'assassiner Arden, enfin suivie du meurtre sauvage et gratuit du héros, contribue à créer l'impression générale de chaos se dégageant de l'œuvre. La trame dramatique de la tragédie, constituée par l'enchaînement apparemment anarchique des péripéties, n'est pas fortuite. Le dramaturge permet au spectateur de prendre le recul nécessaire pour percevoir l'effet artistique ordonnant l'ensemble et lui conférant une dimension esthétique. Le chroniqueur journalistique fournit le matériau brut de départ à l'auteur. Mais son objectif n'allait pas dans le sens de celui du dramaturge, seul organisateur et maitre d'œuvre : ce dernier dépouille la simple chronique du caractère gratuit de la vie quotidienne, pour donner naissance, par le jeu de la création littéraire, à une œuvre d'art.

\section{Jeu des armes, jeu de chasse et jeu esthétique}

L'auteur d'Arden of Faversham joue à mélanger le comique et le tragique. Les deux exécutants du projet meurtrier d'Alice, Blackwill et Shakebag, fascinés par le jeu des armes, sont ridicules dans leurs fanfaronnades : "You were best swear me on the intergatories / How many pistols I have took in hand » (ix.6-7). L'indigence des sentiments humains est palliée par l'abondance d'un langage orienté sur le maniement de l'artillerie de guerre :

\footnotetext{
25 La notion de mouvement désordonné préside, mais sur le mode dérisoire, à la structure de la comédie Gammer Gurton's Needle. La navette, c'est celle des domestiques que Gammer envoie un peu partout, au hasard, chercher l'objet perdu. Quant à l'aiguille, elle suggère, par sa propre nature, un jeu de va-et-vient permanent. Elle n'est que la projection concrète du désarroi de l'héroïne qui n'a plus goût à rien et se sent perdue : " Alas, the more ich [I] think on it, my sorrow waxeth double / My goodly tossing [that moves to and fro quickly in sewing] spurrier's nee'le chave [I have] lost ich wot not [do not know] where. »(II.iv.9-10). Comme chez Arden, le chagrin se mélange à un sentiment d'égarement. Gammer Gurton's Needle, 1552-1563, éd. Charles Whitworth, éd. The New Mermaids, A. \& C. Black, Londres, 1997.
} 
BLACK WILL. Or whether I love the smell of gunpowder, Or dare abide the noise the dag will make, Or will not wink at flashing of the fire.

(ix.8-10)

Le plaisir verbal de Shakebag affleure dans la construction anaphorique de ces vers. Presque toutes les activités sensorielles sont concernées : le toucher (v. 7), l'odorat (v. 8), l'ouïe (v. 9), et la vue (v. 10). Mais, ironiquement, les sens peuvent s'épanouir uniquement parce que leur activité s'organise autour du jeu des armes, instruments de mort, mais objets de fascination pour Black Will. Pourtant, au début de l'ère moderne, l'usage des armes n'était pas systématiquement mal perçu. Dans l'introduction de son ouvrage dialogique, Toxophilus, The Schole of Shootinge, Roger Ascham annonce son désir de ne pas brusquer le lecteur: "My mind is ... to hurte or displease no man, ... shewynge howe fit shootyng is for all kyndes of men, howe honest a pastyme for the mynde, howe holsome an excercise for the bodye... ${ }^{26}$. » Il défend une activité louable, répète-t-il plusieurs fois ${ }^{27}$. L'envolée lyrique de Shakebag (ix.6-10) rappelle partiellement le développement de l'érudit Philologus. Dans le texte d'Ascham, il dit à Toxophilus, amateur des activités associées au maniement des armes : « ...the tunge, the nose, the handes and the feete be no fytter membres, or instrumentes for the body of a man, then is shotinge for the hole bodye of the realme ${ }^{28}$. » Comme Shakebag, Philologus fait référence à certaines parties du corps en relation avec les activités sensorielles de l'homme. Mais sa démonstration est plus élaborée : le personnage met en parallèle l'être humain, dans sa dimension microcosmique, avec le macrocosme. L'activité du tir est salutaire pour l'ensemble du royaume, démontre-t-il. Toxophilus partage ce point de vue, et il le développe plus amplement par la suite. Le maniement des armes fournit aux jeunes gens du pays un exutoire sain, affirme-t-il : « Euen so shulde the teaching of youth to shote, not only make them shote well, but also plucke awaye by the rootes all other desyre to noughtye pastymes, as disynge, cardyng, ... which ... are vsed euery where, to the great harme

\footnotetext{
${ }^{26}$ Roger Ascham, Toxophilus, The Schole of Shootinge, 1545, dans English Works of Roger Ascham, éd. William Aldis Wright, C.U.P., 1904, Rpt. 1970, p. 1-119, p. xiii.

27 Ibid., p. 10 et 22.

28 Ibid., p. 32.
} 
of all youth of this realme ${ }^{29}$. » En utilisant intelligemment les armes, la jeunesse du royaume se détourne des jeux dévoyés, susceptibles de la corrompre. Ce type de considération honorable ne concerne certainement pas Shakebag: pour lui, l'arme est un instrument permettant à son utilisateur d'affirmer une volonté de puissance stupide et destructrice. Le malandrin tire sa force vitale à travers sa relation avec les jouets de mort: "I have took more purses in this Down / Than e'er thou handlest pistols in thy life » (v. 12-13). L'animosité et la combativité, attisée par la moindre parole malencontreuse, semblent être les seules valeurs reconnues pas ces personnages. Les deux assassins sont de simples exécutants, et, malgré leurs fanfaronnades, ils sont bien les seuls à être convaincus de jouir d'une liberté d'action. Les tueurs, restreints à une action violente mais aveugle, échouent toujours. L'auteur s'amuse, dans les scènes où ils interviennent, à créer une détente comique. Cependant le but de l'horreur comique est de faire ressortir la monstruosité de l'héroïne meurtrière.

Le jeu de la chasse constitue une autre variante du divertissement cruel. Mais, dans l'univers de Two Lamentable Tragedies, il n'y a pas de contre-pied comique. Quelques instants après la découverte par Allenso du cadavre de son cousin Pertillo, apparaît le duc de Padoue. Il décrit une scène de chasse au lièvre :

DUKE. How now my Lords, was't not a gallant course?

Beleeve me sirs, I never saw a wretch,

Make better shift to save her little life :

The thickets full of buskes and scratching bryers,

A mightie dewe, a many deepe mouth'd hounds,

Let loose in every place to crosse their course,

And yet the Hare got cleanly from them all :

I would not for a hundred pound in faith,

But that she had escaped with her life,

For we will winde a merry hunters horne,

And start her once againe to morrow morne.

(xiv.131-141)

L'évocation du lièvre poursuivi est une reformulation métaphorique de la situation de l'orphelin assassiné par les hommes de main de son oncle Fallerio. Le motif du lièvre désorienté revêt une

${ }^{29}$ Ascham, op. cit., p. 58 . 
valeur esthétique, contribuant à l'unité structurelle de l'œuvre et renforçant la perspective tragique. La situation de Pertillo renvoie à l’image du lièvre chassé, symbole de la victime, opprimée par les plus forts. Two Lam entable Tragedies présente une vision sombre, puisque les rapports entre les individus seraient fondés sur le lien entre le bourreau et sa victime. Ainsi les relations humaines seraient réduites à un jeu de chasse sinistre, circonscrit dans un espace clos. Les dominants trouveraient leur plaisir en traquant les plus faibles, condamnés à perdre, car enfermés dans un univers sans issue. Le passage où apparaît le duc devient alors une mise en abyme de la pièce. Encastré dans la quatorzième scène, ce morceau pourrait sembler un hors-d'œuvre, mais, les correspondances métaphoriques entre l'animal poursuivi et les personnages victimes le rattachent directement au reste de la scène, et même à l'ensemble de la tragédie. Le lièvre harcelé est un double métaphorique du jeune orphelin Pertillo, assassiné à l'instant même (v. 119, didascalie). Mais Allenso, qui perçoit en son cousin un second soi-même (7, 77 et $84-88)$, est aussi en quelque sorte le double de Pertillo. Ce dernier rappelle le rôle d'éducateur tenu par son cousin dans divers domaines: "...he taught me how to say my prayers, / To ride a horse, to start the fearfull Hare... » (xii.15-16). Ces dernières paroles introduisent discrètement le motif du lièvre chassé. Celui-ci apparaît ensuite dans la quatorzième scène ; mais ce ne sont plus les deux cousins qui lèvent le lièvre. Non sans ironie, ils se trouvent placés, au contraire, dans la position de victimes ou de proies. Le procédé des jeux de miroir permet alors de mettre les personnages en correspondance par spécularité métaphorique. Un personnage renvoie donc à un autre, et ainsi le spectateur n'est pas surpris d'entendre Fallerio désigner son fils, devant Sostrata, par une expression pleine de mépris, "your harebrainde sonne " (xxi.50), avant de compléter sa description par des termes empruntés à un bestiaire avilissant (v. 51). Les personnages d'Allenso et de Pertillo se reflètent, et l'image du lièvre souligne ce rapprochement. Le motif esthétique du lièvre chassé, dans le bref passage inséré dans la quatorzième scène, informe sur la technique du dramaturge. Yarington procède à un jeu d'imbrications, d'analogies, d'échos et de 
correspondances. L'auteur présente au spectateur une variété d'images et de motifs : ils se reflètent et se font écho, ou au contraire s'opposent. La tirade du duc (xiv.131-141), décrivant la course éperdue du lièvre (v. 131), faite de bonds et de feintes (v. 133), tandis que les chiens de la meute (v. 135), lâchés dans tous les coins de l'espace, sont amenés à se croiser au cours de leur traque (v. 136), suggère l'idée d'une danse macabre, quadrille cruel au cours duquel les exécutants passent d'un coin à son opposé, en opérant une traversée. Quelques vers plus bas, Vesuvio évoque le parcours au multiples courbes dessinées par le lièvre en fuite: "But Pendragon gave the Hare more turnes » (v. 146). Tout ce lexique rend compte du jeu entre chasseur et chassé, des ruses mises en œuvre pour échapper au traquenard, et de l'art de l'esquive. La tirade est une vignette insérée dans la trame dramatique ; on peut la rapprocher du court récit relaté par Vénus dans Venus and Adonis. La déesse raconte à Adonis une fable initiatique (v. 674-708) sur la vie, ou plutôt la survie du faible, symbolisé aussi par le lièvre terrorisé poursuivi par les chiens : "Uncouple at the timorous flying hare... » (v. 675). Le lièvre du poème de Shakespeare, comme celui de la pièce de Yarington, n'est qu'une misérable victime, qualifiée de "poor wretch» par Vénus (v.680), terme utilisé par le duc, dans Two Lam entable Tragedies. L'animal survit uniquement grâce à sa capacité d'inventer des stratégies pour dérouter son prédateur. Comme chez Yarington, le lièvre du poème narratif décrit, dans sa fuite, d'innombrables courbes et de multiples zigzags: "Mark/ How he outruns the wind, and with what care / He cranks and crosses with a thousand doubles. »(v. 680-682). Dans la tragédie de Yarington les déplacements du lièvre, tout comme ceux des chiens, sont appréhendés dans leur caractère réitératif. En effet, la tirade s'achève sur l'idée de recommencement, puisque le duc déclare reprendre la chasse le lendemain (v. 141). L'épisode de la traque du lièvre, mis en abyme dans la quatorzième scène, peut se lire comme une métaphore de l'art du dramaturge : son œuvre est un jeu esthétique construit sur le motif du quadrille. Yarington conduit le spectateur d'un lieu scénique à l'autre, puisque l'action se passe en alternance en Angleterre et en Italie. Cette navette géographique reproduisant un schéma parallèle dans chacun 
des deux pays, tout en croissant les deux intrigues d'une scène à l'autre, est un jeu de chassé-croisé artistique. L'œuvre de Yarington donne à voir un monde kaléidoscopique aux nombreuses facettes où miroite une variété d'images, de motifs, d'échos et de reflets.

Il est souvent question de jeu dans les œuvres dramatiques de l'époque élisabéthaine et jacobéenne. Dans les moralités, le jeu peut être risqué, mais la conclusion est toujours optimiste parce que le pardon, découlant du repentir du héros, lui permet d'accéder au salut après la mort. S'il existe de nombreuses analogies entre les moralités et A Yorkshire Tragedy, notamment dans le motif du " fils prodigue », il faut néanmoins souligner une divergence essentielle. La tragédie domestique anonyme s'achève sur une note très sombre. Le héros de $A$ Yorkshire Tragedy, en dépit de son désir de repentir (x.47-48 et 54), est inéluctablement damné. Le jeu l'a conduit à sa perte, à celle de sa famille et au meurtre de ces enfants. Dans Arden of Faversham, le jeu est macabre et gratuit. Le divertissement n'est plus. Seul demeure le vide dans un univers absurde où le Dieu réconfortant des moralités semble absent. Mais le motif se pare aussi d'une valeur métaphorique et théâtrale, dimension esthétique absente des moralités. Le jeu esthétique de la création artistique est peut-être la seule récréation que s'offrent les dramaturges qui, par ailleurs, brossent un univers sombre et sans merci où le divertissement, parfois joyeux, souvent cruel, ne peut être, au mieux, que de bien brève durée. 